\title{
Optimal Modelling and Estimation of a Class of Dynamic Signals in Big Data System Analysis
}

\author{
Xiaonan Xiao
}

\author{
Xiamen University Tan Kah Kee College, Zhangzhou, Fujian, China \\ *Corresponding author.Email: xiaoxn@xujc.com
}

\begin{abstract}
Taking the time series analysis of complex stochastic processes as the main line, this paper makes an in-depth study on a kind of random dynamic information flow widely existing in science and technology, and obtains the nonlinear dynamic model and its optimal control and prediction method under the random century series. In order to further study earthquake prediction, meteorological and financial data analysis, incomplete observation of time series, and outlier processing provide an effective mathematical processing means and method.
\end{abstract}

Keywords: Big data system analysis, ynamic signal, Mplex stochastic process, Telligent computing, timization modeling

\section{INTRODUCTION}

Complex random process time series is a very important method and research hotspot in the discipline of probability and statistics. They are widely used in many fields, such as earthquake research, hydrometeorology, economy and finance, signal processing, mechanical vibration and so on [1-3]. Facing the rapid development of social economy and the urgent demand for information development and technology in various fields of science and technology in the new century, this paper will use the methods of complex random process time series analysis to deeply analyze and study the important random dynamic information flow of science and technology, with innovative thinking, advanced methods, reliable conclusions and remarkable results, This paper provides an effective

$$
\begin{gathered}
\alpha_{S+1}=\theta_{1} \alpha_{S}+\theta_{2} \sigma_{S}+\left(d D^{*}+c_{1} i_{S} E^{*}\right)\left(D D^{*}+E_{1} i_{1} E^{*}\right)^{+}\left(\sigma_{S+1}-E_{1} \alpha_{S}-E_{2} \sigma_{S}\right) \\
i_{s+1}=c_{1} i_{s} c_{1}^{*}+d d^{*}-\left(d D^{*}+c_{1} i_{s} E_{1}^{*}\right)\left(D D^{*}+E_{1} i_{1} E_{1}^{*}\right)\left(d D^{*}+c_{1} i_{s} E_{1}^{*}\right)^{*}
\end{gathered}
$$

The equation is based on the initial condition:

$$
\alpha_{0}=E\left(\beta_{0} \mid \sigma_{0}\right), i_{0}=E\left[\beta_{0}-\alpha_{0}\right]\left[\beta_{0}-\alpha_{0}\right]^{*}
$$

To solve According to normal correlation theorem

$$
\begin{aligned}
& \left.\alpha_{0}=\operatorname{cov} \beta_{0}, \sigma_{0}\right) \cot \left(\sigma_{0}, \sigma_{0}\right) \sigma_{0} \\
& \left.\left.\left.i_{0}=\operatorname{cov} \beta \beta_{0}, \beta_{0}\right)-\operatorname{cov} \beta_{0}, \sigma_{0}\right) \operatorname{cov}\left(\sigma_{0}, \sigma_{0}\right) \operatorname{cov} \beta \beta_{0}, \sigma_{0}\right)
\end{aligned}
$$


Since $\alpha_{\mathrm{s}}=E\left(\xi_{t} \mid \mathrm{F}{ }_{\mathrm{s}}^{\beta}\right)$ linearly depends $\sigma_{0}, \cdots, \sigma_{s}$, in the case of Gauss process $X_{s}=\left[\beta_{s}, \sigma_{s}\right]$, constitute $\beta_{\mathrm{s}}$ the best linear estimation problem according to $\sigma_{0}, \cdots, \sigma_{\mathrm{s}}$ the solution by the equation (1), (2) is given.

For the general case, the random vector is provided $(W, \theta)$ with $E\left(W^{2}+\theta^{2}\right)<\infty$, and $(W, \theta)(\theta, W)$ with the same front two moments of Gauss vector,

ie $E W^{t}=E W^{t}, E^{t} \theta=E \theta^{t}, t=1,2$.if $\mathrm{i}(\mathrm{c})$ is $Q_{-\Pi . H .}$ making

$$
i(W)=E(\theta \mid W)
$$

$l(\theta)$ is the best linear estimation of the amount by $W$ and $\theta$ in the mean-square sense, and $\operatorname{Ei}(\theta)=E W$.

When the vector is $W=\left(W_{1}, \cdots, W_{j}\right), \quad \theta=\left(\theta_{1}, \cdots, \theta_{i}\right)$, the above conclusion remains valid.

And then in the containable control, the best control $y=\left(y_{s}\right), 0 \leq s \leq S$ is decided by the formula below:

$$
y_{s}=-Q^{-1}(s) a^{*}(s) F(s) \alpha_{t} 0 \leq s \leq S
$$

where the nonnegative definite symmetric matrix $F(s)=\left\|F_{i j}(s)\right\| \quad 0 \leq s \leq S$ of the stage

of $(r \times r)$, is the answer to the following Riccati equation:

$$
\begin{aligned}
& -\frac{d \eta(s)}{d s}==a^{*}(s) p(s)+p(s) a^{*}(s)+ \\
& q(s)-p(s) C(s) S^{-1}(s) C^{*}(s) \eta(s), \\
& \eta(s)=t
\end{aligned}
$$

while vector $\alpha_{s}$ is determined by the equation group below:

$$
\begin{aligned}
& d \alpha_{s}=\left[d(s) y_{s}+b(s) \alpha_{s}\right] d s \\
& +i_{s} E^{*}(s)\left(D(s) D^{*}(s)\right)^{-1}\left[d \sigma_{s}-E(s) s_{s} d s\right], \\
& \alpha_{0}=\alpha_{0}=R \beta_{0} \\
& i_{s}=C(s) s_{s}+i_{s} c^{*}(s)+d(s) d^{*}(s) \\
& -i_{s} E^{*}(s)\left(D(s) D^{*}(s)^{-1} E(t) l_{s},\right. \\
& \qquad i_{0}=\operatorname{cov}\left(\beta_{0}, \beta_{0}\right) \\
& \quad X(y, M)=Q(0)+\alpha_{0}^{*} Q(0) \alpha_{0} \\
& \quad+\alpha_{Q} \int_{0}^{S}\left[\eta^{1 / 2}(s) i_{s} \eta^{1 / 2}(s)+l^{1 / 2} i_{T} l^{1 / 2}\right] d s
\end{aligned}
$$

where

$$
\eta(s)=\int_{s}^{s} \sum_{t, r=1}^{j} Z_{t r}(\alpha) \eta_{t r}(\alpha) d \alpha
$$

while $Z_{t r}(s)$ is the element of the matrix

$$
Z(s)=i^{*} E(s)\left[D(s) D^{*}(D)\right]^{-1} E(s) l_{s} .
$$

\section{OPTIMIZATION MODELING ANALYSIS AND INTELLIGENT COMPUTING}

(1) Allowed spectrum expression generalized stationary process $S(s), s=0, \pm 1, \pm 2, \cdots$, is part of a component of the n-dimensional generalized stationary process $\left(S_{1}(s), \cdots, S_{n}(s)\right)$ following recursion equations, $S_{1}(s)=S(s) \quad S_{n}(s+1)$ $S_{r}(s+1)=S_{r+1}(s)+\theta_{r} H(s+1), r=1,2, \cdots, n-1$,

$$
=-\sum_{r=0}^{n-1} W_{r} S_{r+1}(s)+\theta_{n} H(s+1)
$$

Process $\quad H(s), s=0, \pm 1, \pm 2, \cdots, \quad$ Available show by (6)

$$
E S_{r}(s) H(s)=0, \quad s<\alpha, \quad r=1,2, \cdots, n
$$

The coefficient $\theta_{1}, \theta_{2}, \cdots, \theta_{n}$ by equation (6) given.

If $S(s), s=0, \pm 1, \pm 2, \cdots$, is a real process, each process $H(s), S_{2}(s), \cdots, S_{n}(s)$ is a real process. 
If $S(s), s=0, \pm 1, \pm 2, \cdots$, is a Gauss process, $H(s), s=0, \pm 1, \pm 2, \cdots, \quad$ is also a Gauss sequence comprising independent random variables.

$$
\text { Set } X_{s}=\left[\beta_{s}, \sigma_{s}\right]=\left[\left(\beta_{1}(s), \cdots, \beta_{j}(s)\right),\left(\sigma_{i}(s), \cdots, \sigma_{s}(s)\right)\right], s=0, \pm, 1 \pm 2, \cdots
$$

for the real general smooth $i+s$ dimensional process, can be expressed as

$$
X_{s}=\int_{-l}^{l} e^{t \mu s} P\left(e^{t \mu}\right) g(d \mu)
$$

Which $P(J)=\left\|P_{l, p}(J)\right\| \quad$ is an $\quad \boldsymbol{m} \times \boldsymbol{n}$ order matrix, $\boldsymbol{m}=j+$ 1 , a fractional rational element

$$
P_{l, p}(K)=\frac{X_{n_{r, p-1}^{(l, p)}}}{Y_{n_{l, p}^{(l, p)}}^{(l, p}}
$$

And $\quad \varphi(d \mu)=\left[g_{1}(d \mu), \cdots, g_{m}(d \mu)\right] \quad$ as $\quad$ a non-related components of the random vector measure,

$$
E g_{t}(d \mu)=0, \quad E\left|g_{r}(d \mu)\right|^{2}=\frac{1}{2 l} d \mu
$$

And assuming the root of the equation $Y_{n_{l, p}}^{(l, p)}(J)=0$ is located in the unit park.

Application process for each of the following lines (1),

$$
X_{q, l, p}(s)=\int_{-l}^{l} e^{t \mu s} P_{l, p}\left(e^{t \mu}\right) g_{q}(d \mu)
$$

Vector $\sigma_{s}=\left(\sigma_{1}(s), \cdots, \sigma_{i}(s)\right)$ and vector $\beta_{i}$ [composed of vector $\beta_{s}=\left(\beta_{1}(s), \cdots, \beta_{j}(s)\right)$ and shaped like $S_{2}(s), \cdots, S_{n}(s)$ all those additional components] after the recurrence equation obtained by simple transformation group

$$
\begin{aligned}
\beta_{s+1}= & c_{1} \beta_{s}+c_{2} \beta_{s}+c H(s+2) \\
& \sigma_{s+1}=E_{1} \beta_{s}+E_{2} \sigma_{s}+B H(s+2)
\end{aligned}
$$

wherein $H(s)=\left(H_{1}(s), \cdots, H_{m}(s)\right) \quad$ is a sequence of non-correlation component of the non-associated vector,

$$
\begin{aligned}
E H_{r}(s)= & 0, \quad E H_{r}^{2}(s)=1 \\
& H_{r}(s)=\int_{-l}^{l} e^{t \mu(s-1)} g_{r}(d \mu)
\end{aligned}
$$

(2) Expression (6) can be exported containing fractional rational spectral density of multidimensional generalized stationary sequence component filter equations. and matrix $\mathrm{C}_{\mathrm{t}}, \mathrm{E}_{\mathrm{t}}, a$ and $A, t=1,2$, that can be obtained directly.

(3) Given $\sigma_{s}, s=0, \pm 1, \pm 2, \cdots$ is a generalized stationary process with $E \sigma_{s} \equiv 0$ and the spectral density is $Z(\mu)=\left|e^{t \lambda}+3\right|^{2} /\left|e^{2 t \mu}+e^{t \mu} / 4+2 / 3\right|^{2}$, in order to determine $\sigma_{s}$ with $\sigma_{0}^{\alpha}=\left\{\sigma_{0}, \cdots, \sigma_{\alpha}\right\}$ in accordance with the best linear estimation (which $s \leq \alpha$ ) in the mean square sense, we need to have structure and spectral density Gauss process. And this process by the equation

$$
\sigma_{s+3}+\frac{1}{4}\left(\sigma_{s+2}+\sigma_{s}\right)=H(s+3)+H(s+2)
$$

By the answer obtained, which is a Gauss random sequence:

$$
E H(s)=0, \quad E H(s) H(\alpha)=\delta(\alpha, s)=s
$$

Now assume $\beta_{s}=\sigma_{s+1}-H(s+1)$, for the random vector $\left(\beta_{s}, \sigma_{s}\right), s=0, \pm 1, \pm 2, \cdots$, can be obtained equations

$$
\begin{gathered}
\beta_{s+2}=-\frac{1}{4} \beta_{s}-\frac{1}{4} \sigma_{s}+\frac{1}{4} H(s+1) \\
\sigma_{s+2}=\beta_{s}+H(s+2)
\end{gathered}
$$

wherein $n_{1}(s, \alpha)=E \quad\left(\begin{array}{l|ll}\eta_{s} & \mid \mathrm{F} & \xi \\ s\end{array}\right) \quad$ and $n_{2}(s, \alpha)=E\left(\beta_{s} \mid \mathrm{F}_{s}^{\beta}\right)$ by having the initial conditions

$$
T_{1}(s, s)=s_{s}, \quad T_{2}(t, t)=l_{s}
$$

equation

$$
\begin{array}{r}
S_{1}(\alpha+2, t)=-\frac{3}{4} S_{1}(\alpha, s)-\frac{1}{4} S_{2}(\alpha, s) \\
S_{2}(\alpha+2, s)=S_{1}(\alpha, s)
\end{array}
$$

definite. then(12)initial condition $S=E\left(\beta_{\alpha} \mid\right.$ $\mathrm{F}_{s}^{\beta}$ ) and $\boldsymbol{l}_{\mathrm{s}}$ also defined by equation 


$$
\begin{gathered}
\alpha_{n+1}=-\frac{3}{4} \alpha_{n}-\frac{1}{4} \sigma_{s}+\frac{2-i_{n}}{4\left(2+l_{n}\right)}\left(\sigma_{n+1}-\alpha_{n}\right) \\
i_{n+1}=\frac{i_{n}}{2+l_{n}}
\end{gathered}
$$

and it is not difficult to prove $\alpha_{0}=0, i_{0}=1$.

Described above, in generalized stationary sequence $\sigma_{s}, s=0, \pm 1, \pm 2, \cdots$, the best linear

$$
\begin{array}{r}
\frac{\partial k(y, s)}{\partial s}+\frac{\partial k(y, s)}{\partial y}=\frac{\partial^{1-l}}{\partial s^{1-l}}\left(\frac{\partial^{2} k(y, s)}{\partial y^{2}}-k(y, s)\right)+e^{y}\left[(2+l) s^{l}+s^{2+l}\right] \\
\begin{array}{l}
0 \leq s \leq 1,0<y<1 \quad k(y, 0)=0, \quad \text { Q } y \leq \\
k(0, s)=s^{1+l}, \quad k(1, s)=e s^{1+l}, \quad 0 \leq s \leq 1,
\end{array}
\end{array}
$$

The precise solution for the initial - boundary value problems of (15) - (17) is $u(\mathrm{y}, \mathrm{s})=e^{y} s^{1+l}$.

Table 1. The maximum error $E_{\infty}$ of numerical methods (8) - (9)

\begin{tabular}{cccc}
\hline$l$ & $\Delta_{s}=\Delta_{y}^{2}=1 / 4$ & $\Delta_{t}=\Delta_{\mathrm{y}}^{2}=1 / 16$ & $\Delta_{s}=\Delta_{y}^{2}=1 / 64$ \\
\hline 0.4 & $2.3804 \times 10^{-3}$ & $4.7963 \times 10^{-4}$ & $3.5735 \times 10^{-5}$ \\
0.7 & $3.7862 \times 10^{-3}$ & $6.8902 \times 10^{-4}$ & $4.3867 \times 10^{-5}$ \\
0.9 & $5.8675 \times 10^{-3}$ & $7.5638 \times 10^{-4}$ & $5.3278 \times 10^{-5}$
\end{tabular}

\section{CONCLUSIONS}

This paper broadens the new ideas of random information analysis, intelligent computing and decision-making technology, and its research theories and methods are widely used. A series of theories, methods and results obtained in this paper will provide reliable mathematical theories and methods for the further study of the optimization and control of random information.

\section{EXPECTATION}

After the unprecedented development of intelligent decision technology in the 20th century, its theories and methods have been further deepened and improved. The vigorous and development of computer technology makes the application of mathematics more direct and extensive, and effectively promotes the development of science, technology and social economy[8-9]. All these are changing people's traditional understanding of Mathematics, At the same time, it also promotes the great changes in the research methods of mathematics. Mathematics, as the basis of science and technology, is breaking through the traditional scope and penetrating into all fields of human knowledge. Mathematical science has become an important factor to promote the progress of human civilization and knowledge innovation. It will more profoundly change the objective appearance and people's understanding of the world. The great development of mathematical theory and application will inevitably lead to profound changes in science and technology [10-12]. Let's join hands and work together to improve the scientific and technological level of all mankind.

\section{REFERENCES}

[1] Liu Z, Wang Y. Handing Constrained Multiobjective Optimization Problems With Constraints in Both the Decision and Objective Spaces [J]. IEEE Transactions on Evolutionary Computation, 2019, 23(5): 870-884.

[2] Mo Jiaqi, Yao Jingsun. A class of Singular perturbed nonlinear reaction diffusion 
equations with two parameters [J]. J Math, 2011, 33(2): 341-346.

[3] Mo Jiaqi. Singularly perturbed asymptotic solutions for higher order semilinear elliptic equations with two parameters $[\mathrm{J}]$. Chinese Annals of Mathematics, 2010, 33A(1): 331-336.

[4] He Q, Wang L. An effective co-evolutionary particle swarm optimization for constrained engineering design problem $[\mathrm{J}]$.Engineering Applications of Artificial Intelligence, 2007, 20(1):88-89

[5] Mo Jiaqi, Liu Shude. Singularly perturbed solution for semilinear reaction diffusion equation with two parameters [J]. Applied Mathematics and Mechanics, 2009, 30(5): 607-612.

[6] Shi Kaiquan, Yao Bingxue. Function S-rough sets and las identification [J]. Science in China Series F: Information Sciences, 2008, 51(5): 499-510.

[7] Ren Y, Lu SP, Xia N M. Remarks on the existence and uniqueness of the solutions to stochastic functional differential equations with infinite delay [J]. Comput Appl Math, 2008, 220: 364-372.

[8] Wang QH. Statistical estimation in partially linear models with covariate data missing at random[J].Annals of the Institute of Statistical Mathematics, 2009, 61:47-84

[9] Yu L, Chen GD, Chu J. Optimal guaranteed cost control of linear uncertain systems: LMI approach [J]. IFAC, 1999, G-2e-21-1: 541-546.

[10] Zhou Minggru, Du Zengji, Wang Guangwa. The Theory of Differential Inequality In Singular Perturbation [M]. Beijing: Science Press, 2012.

[11] Liu Shude, Lu Shiping, Yao Jingsun, Chen Huai-jun. Singular Perturbation Boundary And Corner Layer Theory $[\mathrm{M}]$. Beijing: Science Press, 2012.

[12] Lin Y F, Du W, Du WL,et al. Multi-objective differential evo-lution with dynamic hybrid constraint handling mechanism[J].Soft Computing, 2019, 23(12):4341-4355. 